\title{
Thixotropic Behavior of Metal-Containing Coordination Polymers: Melt Viscosity of Neutral Aliphatic Polyesters With Zn Carboxylates
}

\author{
G. LIU, ${ }^{1}$ B. WHITE, ${ }^{2}$ I. VANCSÓ-SZMERCSÁNYI, ${ }^{1}$ and G. J. VANCSO ${ }^{1, \star}$ \\ 'University of Twente, Faculty of Chemical Technology, P.O. Box 217, NL-7500 AE Enschede, The Netherlands; \\ ${ }^{2}$ Ortech Corporation, 2395 Speakman Drive, Mississauga, Ont. L5K 1B3, Canada
}

\begin{abstract}
SYNOPSIS
The viscosity behavior of polymer melts containing complexes formed between the neutralized polyester poly (diethylene glycol-co-succinic acid) and $\mathrm{Zn}$ acetates is discussed. The melt viscosity of these materials increases with the concentration of metal ions, and shows strong thixotropy and shear thinning. This behavior is attributed to the formation of coordination bonds between the electron donor groups within the polyester chain, and empty coordination sites of the various $\mathrm{Zn}$ acetate salts. The coordination complexes were obtained in situ in the polymer melt, which contains well-dispersed $\mathrm{ZnO}$, by adding an equimolar amount of $\mathrm{CH}_{3} \mathrm{COOH}$. It is proposed that the shear applied to the polymer melt destroys the polar network of the coordination polymer at a rate that is greater than the rate of reformation of the coordination bonds for the sample returning back to equilibrium, following a shear deformation. (c) 1996 John Wiley \& Sons, Inc.

Keywords: coordination polymers $\bullet$ melt viscosity $\bullet$ thixotropy $\bullet$ polyesters $\bullet$ telechelic networks
\end{abstract}

\section{INTRODUCTION}

The viscosity of non-Newtonian fluids depends on their previous kinematic history (e.g., the shear rate) ${ }^{1,2}$ In a simple case, the shear rate is a function of the shear stress only, and does not depend on time. For more complex systems, the relationship between shear stress and shear rate may depend on how long the fluid has been sheared. For thixotropic fluids, the shear stress decreases with time if the fluid is sheared at a constant rate. This decrease is a result of the breakdown of structural links between the molecules of the fluid. If the shear rate decreases, the structural links in the thixotropic fluid will build up again, but at a different rate. If the rate of the structural breakdown and the rate of re-formation of the links are the same, then the fluid does not show time-dependent behavior.

\footnotetext{
* To whom correspondence should be addressed.

Journal of Polymer Science: Part B: Polymer Physics, Vol. 34, 277-282 (1996) (C) 1996 John Wiley \& Sons, Inc. CCC 0887-6266/96/020277-06
}

The rheological properties of coordination polymers formed in the reaction of low molecular weight polyesters with $\mathrm{MgO}^{3}$ and $\mathrm{ZnO}^{4}$ has been the subject of extensive previous work. In polyesters containing carboxylic chain-terminating groups, various complexes can form between the terminal $\sim \mathrm{COOH}$ and the metal oxides. ${ }^{5,6}$ As a result, the linear polymer chains will link, forming an ionic network. Depending on the concentration of metal ions $\left(\mathrm{Me}^{2+}\right)$ in the sample, the viscosity increases over several orders of magnitude. In the polyester melt, different ligands can coordinate with the central metal ions, including oxygen atoms in $\sim \mathrm{OH}$, in $\sim \mathrm{COOH}$, or in the ester groups. Both the total number of the coordination bonds and the relative amount of different types of these bonds (which is a function of the concentration of $\mathrm{Me}^{2+}$ ) influence the activation energy of the viscous flow. The activation energy shows a marked increase with increasing amount of $\mathrm{Me}^{2+}$.

If the terminal $\sim \mathrm{COOH}$ groups are neutralized in the polyester prior to the introduction of metal oxides, no ionic network can form. However, upon introducing appropriate amounts of monofunctional 
organic acids into the mixtures of the polyester [which contain well-dispersed metal oxide (MeO) powders] the acid will react with the metal oxide, forming various types of small-molecule acetates. ${ }^{4}$ In this reaction, coordination bonds will form between the electron donor groups of the neutral polyester chain and the acetates. The electron donors encompass the chain-internal carbonyls of the ester groups and the chain-terminating $\sim \mathrm{OH}$ groups. In this work, we report on our studies regarding the time-dependent viscosity behavior of such complexed neutralized polyesters.

\section{EXPERIMENTAL}

\section{Sample Preparation}

Saturated polyesters of diethylene glycol (DEG) and succinic acid (SA) were synthesized by melt polycondensation at $180^{\circ} \mathrm{C}$ using a molar ratio of DEG/ $\mathrm{SA}$ of 1.02 in the usual fashion. ${ }^{7}$ The extent of the reaction was monitored by measuring the numberaverage molar mass by end-group titration using acid numbers. ${ }^{8}$

In previous studies, it has been shown that $\mathrm{MeO}$ $(\mathrm{Me}=\mathrm{Zn}$ or $\mathrm{Mg})$, if added to not-neutralized poly(DEG-SA), forms complexes with the chainterminating carboxylic groups. ${ }^{3,5,6}$ The structure of these complexes depends on the molar ratio of $\left[\mathrm{Me}^{2+}\right]$ vs. $\left[\sim \mathrm{COO}^{-}\right]$. At the molar ratio of ca. 0.5 , primarily carboxylate complexes (Scheme 1) form, whereas at one equimolar ratio the formation of carboxylato-hydroxo complexes become favorable (Scheme 2):

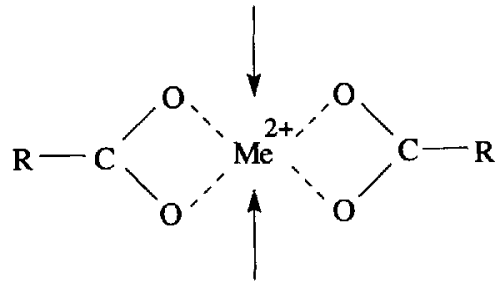

Scheme 1. The preferred complex structure formed between carboxylic groups and a bivalent metal ion at a molar ratio of $\mathrm{Me}^{2+} / \mathrm{COO}^{-}$of ca. $0.5\left(\mathrm{Me}^{2+}=\mathrm{Zn}^{2+}\right.$ or $\left.\mathrm{Mg}^{2+}\right)$.

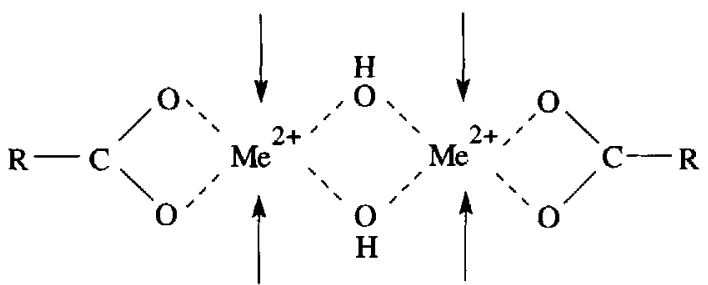

Scheme 2. The preferred complex structure formed between carboxylic groups and a bivalent metal ion at a molar ratio of $\mathrm{Me}^{2+} / \mathrm{COO}^{-}$of ca. $1.0\left(\mathrm{Me}^{2+}=\mathrm{Zn}^{2+}\right.$ or $\left.\mathrm{Mg}^{2+}\right)$.
Complexes with similar structures may also form in reactions of $\mathrm{MeO}$ with low molar mass monofunctional organic acids (e.g., $\mathrm{CH}_{3} \mathrm{COOH}$ ). It is expected that these carboxylate complexes, when present in a neutralized polyester network, will form donor-acceptor bonds with negatively polarized groups of the polyester chain. In the presence of both nonneutralized $\sim \mathrm{COOH}$ chain ends and $\mathrm{CH}_{3} \mathrm{COOH}$, complexes with both types of carboxylic groups will form with $\mathrm{MeO}$. To study the influence of the complexes formed with only low molar mass organic acids and metal oxides on the viscoelastic properties, neutralization of the polyester chainterminating $\sim \mathrm{COOH}$ groups is necessary.

Neutralized poly(DEG-SA) samples were prepared by adding an excess of diethylene glycol into poly(DEG-SA) samples $\left(M_{n} \sim 1000 \mathrm{~g} / \mathrm{mol}\right)$. This reaction was performed at $180^{\circ} \mathrm{C}$ for ca. $10-15 \mathrm{~h}$. During the reaction, end-group titration was utilized to estimate the amount of the remaining carboxylic chain ends. The goal was to obtain neutralized polyesters with alcoholic groups $(\sim \mathrm{OH})$ at both chain ends. When the mole fraction of the remaining carboxylic chain ends was less than $0.1 \mathrm{~mm}$ per gram sample, the reaction was considered to be complete. An oil vacuum pump was then used for another $2 \mathrm{~h}$ to remove the condensed water and excess diethylene glycol from the neutralized polymer.

Polyester complexes containing $\mathrm{Zn}$ acetates were prepared by adding equimolar amounts of a fine powder of $\mathrm{ZnO}$ (Aldrich 25160-7, purity > 99\%) and $\mathrm{CH}_{3} \mathrm{COOH}$ to neutralized poly(DEG-SA) as follows. First $\mathrm{ZnO}$ was evenly dispersed in the poly(DEGSA) melt at room temperature. This step was followed by a thorough mixing with acetic acid, which was added dropwise to the reaction mixture. During this mixing, the heat released by the neutralization reaction of $\sim \mathrm{COOH}$ with $\mathrm{ZnO}$ warmed up the reactants slightly. The resulting slurry was then placed in a hot-air oven and kept at $70^{\circ} \mathrm{C}$ for a period of between $48 \mathrm{~h}$ and 5 days. By choosing the molar ratio of $\mathrm{CH}_{3} \mathrm{COOH}$ and $\mathrm{ZnO}$ equal to 1 , predominantly carboxylato-hydroxo complexes (Scheme 2) would form.

In the neutralized poly(DEG-SA) system, the molar ratio of the carboxylato-hydroxo complex to polyester was varied. Samples were identified by the average number of $\mathbf{Z n}^{2+}$ species per polyester chain.

\section{Measurement of the Melt Viscosity}

The steady shear melt viscosity of polyester samples was measured with a Brookfield programmable rheometer Model DV-III. The viscometer measures the torque required to rotate an immersed element 
(the spindle) in a fluid. The spindle is driven by a synchronous motor with a calibrated spring. By utilizing a multiple (four or eight)-speed transmission and interchangeable spindles, a variety of viscosity ranges can be measured. The calibration of the rheometer was verified by comparing the quoted viscosity values of a standard silicon oil (HT30'000, Brookfield Eng. Labs. Inc.) at three temperatures with measured values. In all cases the agreement was better than $\pm 2 \%$.

Melt viscosity experiments involved selecting four different spindle speeds, and recording the viscosity values. For thixotropic materials, the measurement of the melt viscosity must follow well-established protocols. The method used to measure sheared and unsheared materials, developed by Asbeck, ${ }^{9}$ and modified by Pierce, ${ }^{10}$ was utilized in our work. To characterize the unsheared samples, the viscometer was started at the lowest speed setting. After at least 10 revolutions, the viscosity value was recorded. The viscometer was then stopped until the recovery of the polymer melts was complete (about $4-5 \mathrm{~h}$ ). The measurement was then started at the next higher speed setting. The procedure was continued at successively higher speeds until the torque reading in Brookfield units exceeded a value of $90 \%$ of the total range accessible. To measure the time-dependent behavior of the sheared samples, the viscometer was started at the highest speed setting. The viscosity was recorded after 10 revolutions. The measurements were then continued at successively lower speed settings until the torque readings in the Brookfield unit were less than $10 \%$ of the total accessible scale. The torque reading in the Brookfield units can be converted to "real" torque expressed in dyne-centimeters by multiplying with the calibrated spring constant. The viscosity, shear stress, and shear rate can be easily calculated from the torque, the geometry of the spindle, and the angular velocity of the spindle. ${ }^{11}$

\section{RESULTS}

The melt viscosity of the complexes of neutralized poly (DEG-SA) with different amounts of $\mathrm{Zn}$ acetates increased with increasing concentration of $\mathrm{Zn}^{2+}$. The melt viscosities of the pure neutralized poly (DEG-SA) and its complexes with $\mathrm{Zn}$ acetates at different temperatures are shown in Table I. The values of the melt viscosity of the samples with $\frac{1}{4}$ and $\frac{1}{2} \mathrm{Zn}^{2+}$ per chain were taken at the shear rate of $0.9 \mathrm{~s}^{-1}$ after 10 revolutions. Due to the limitations of the viscosity range accessible by the Brookfield viscometer used, no viscosity values are given for the sample with $\frac{1}{4} \mathrm{Zn}^{2+}$ per chain at $70^{\circ} \mathrm{C}$, and for the sample with $\frac{1}{2} \mathrm{Zn}^{2+}$ per chain at $30^{\circ} \mathrm{C}$. Viscometry had to be performed at these two different temperatures for the two different $\mathrm{Zn}^{2+}$ concentrations to allow us to remain within the experimentally accessible viscosity range of the Brookfield setup.

Melt viscosities of the complexed poly (DEG-SA) samples are not constant, but decrease with time when subjected to a constant shear rate. Figure 1 is the plot of the melt viscosity vs. time for the complexed neutralized poly (DEG-SA) including $\frac{1}{4} \mathrm{Zn}^{2+}$ per polyester chain at $30^{\circ} \mathrm{C}$. The shear rate is 5.5 $\mathrm{s}^{-1}$, and the first value of the melt viscosity was obtained after 10 revolutions. A large decrease in the melt viscosity was observed in the first $5 \mathrm{~min}$, after which the observed value gradually leveled off. This indicates that the material develops a timedependent, or thixotropic, behavior.

Experiments showed that the melt viscosity of unsheared neutralized poly(DEG-SA) complex containing $\frac{1}{4} \mathrm{Zn}^{2+}$ per chain (measured at $30^{\circ} \mathrm{C}$ ) is different from that of the sheared sample (see Fig. 2 ). The two straight lines in Figure 2 were calculated using the Casson-Asbeck equation ${ }^{9,10,12}$

$$
\eta^{1 / 2}=\left(\eta_{\infty}\right)^{1 / 2}+\left(\tau_{0}\right)^{1 / 2} \dot{\gamma}^{1 / 2}
$$

where $\eta_{\alpha}$ is the limiting viscosity at infinite shear rate, which represents the condition where all structures have been broken down by deformatic.., and $\tau_{0}$ is the Casson yield stress. The corresponding straight lines have different values of slopes, but have essentially the same intercept with the $y$-axis. The calculated results, according to the linear least squares fits, showed that the limiting melt viscosities, $\eta_{\infty}$, are 4870 and 4790 centipoises for unsheared and sheared samples, respectively. The values obtained for the yield stresses, $\tau_{0}$, of these samples from the slopes were 204 and $520 \mathrm{dyn} / \mathrm{cm}^{2}$, respectively. (The regression coefficients for the corresponding fits were all in the range of $r^{2}>0.99$, thus the correlation of the data with the Casson-Asbeck

Table I. Melt Viscosities (in Centipoise) of Neutralized Poly(DEG-SA) and its Complexes With Zn Acetates at the Shear Rate of $0.9 \mathrm{~s}^{-1}$ and at Different Temperatures

\begin{tabular}{lcc}
\hline & \multicolumn{2}{c}{ Temperature } \\
\cline { 2 - 3 } Sample ID & $30^{\circ} \mathrm{C}$ & $70^{\circ} \mathrm{C}$ \\
\hline Neutralized poly(DEG-SA) & 7000 & 320 \\
Complex with $1 / 4 \mathrm{Zn}^{2+} /$ chain & 8750 & - \\
Complex with $1 / 2 \mathrm{Zn}^{2+} /$ chain & - & $1.00 \times 10^{5}$ \\
\hline
\end{tabular}




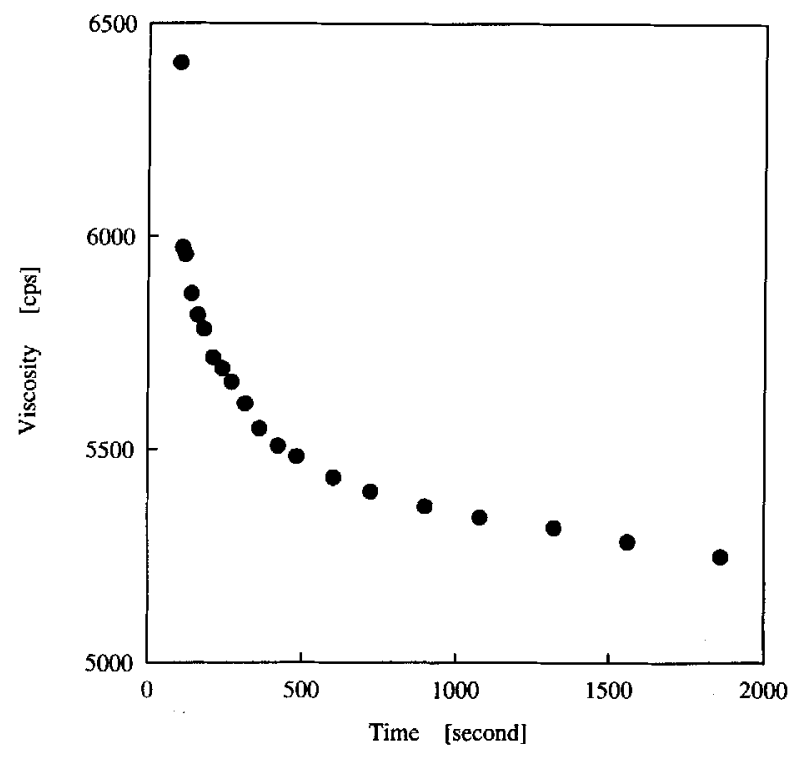

Figure 1. Plot of the melt viscosity, $\eta$, against the duration of shear of the neutralized poly(DEG-SA) complex with $\frac{1}{4} \mathrm{Zn}^{2+}$ per chain at a constant shear rate of $5.5 \mathrm{~s}^{-1}$ at $30^{\circ} \mathrm{C}$.

equation is excellent.) It can be seen that the melt viscosities of unsheared and sheared samples reached, to a good approximation, a common limiting value at an infinite shear rate, regardless of the shear history. This type of behavior indicates a homogeneous melt structure. ${ }^{10}$ The $\tau_{0}$ yield values

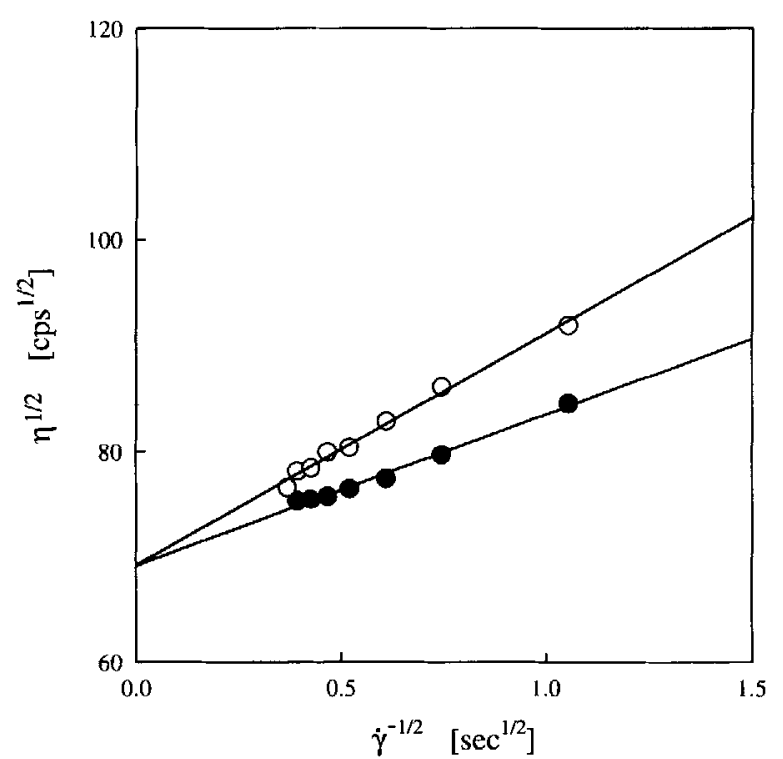

Figure 2. Casson plot of the square root of the viscosity, $\eta^{1 / 2}$ vs. (shear rate) $)^{-1 / 2}, \dot{\gamma}^{-1 / 2}$, of the neutralized poly(DEGSA) complex with $\frac{1}{4} \mathrm{Zn}^{2+}$ per chain at $30^{\circ} \mathrm{C}$. Open circles: unsheared sample; filled circles: sheared sample. The straight lines correspond to linear least squares fits. are, however, sensitive to the shear history, as the unsheared sample has a higher yield value than the sheared one. This decrease of the $\tau_{0}$ value is a result of the breakdown of structural linkages between macromolecules during shear. As can be seen in Figure 3 , the neutralized poly (DEG-SA) complex with $\frac{1}{2} \mathrm{Zn}^{2+}$ per chain gave a qualitatively similar result. As mentioned, the melt viscosity measurement of this sample was performed at $70^{\circ} \mathrm{C}$ due to the limitations of the viscometer used. The values of $\eta_{\infty}$ are $1.50 \times 10^{4}$ and $1.40 \times 10^{4}$ centipoises; the values of $\tau_{0}$ are $2.06 \times 10^{4}$ and $1.64 \times 10^{4} \mathrm{dynes} / \mathrm{cm}^{2}$ for unsheared and sheared samples, respectively. Finally we must mention that the use of the Casson-Asbeck equation allows us to plot our data only in a convenient way and is not used to interpret and describe, at the molecular level, the formation of the ionicpolar network.

Figure 4 shows the double logarithmic plot of shear stress, $\tau$, and shear rate, $\dot{\gamma}$, for unsheared poly (DEG-SA) samples with $\frac{1}{4}$ and $\frac{1}{2} \mathrm{Zn}^{2+}$ per chain. The measurement temperatures were $30^{\circ} \mathrm{C}$ for the sample with $\frac{1}{4} \mathrm{Zn}^{2+}$ per chain, and $70^{\circ} \mathrm{C}$ for the sample with $\frac{1}{2} \mathrm{Zn}^{2+}$ per chain, respectively. The straight lines were fit according to the power law relationship, $\tau=\kappa \dot{\gamma}^{\nu}$, where $\nu$ is the power index, and $\kappa$ is a constant. The results show that the power index $\nu$ is less than 1 for both materials ( $\nu=0.88$ for the sample containing $\frac{1}{4} \mathrm{Zn}^{2+}$ per chain at $30^{\circ} \mathrm{C}$, and $\nu=0.45$

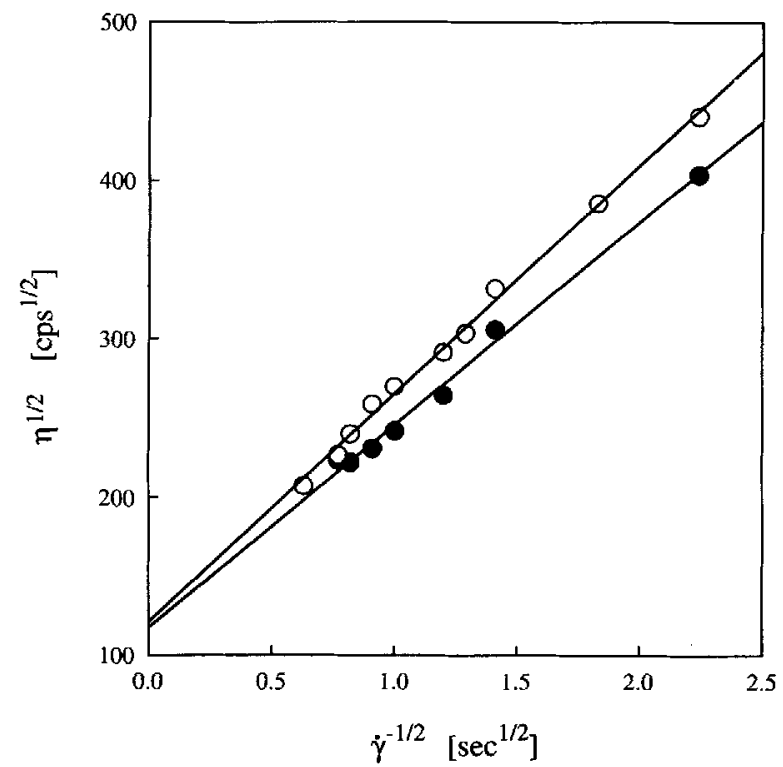

Figure 3. Casson plot of the square root of the viscosity, $\eta^{1 / 2}$, vs. (shear rate) $)^{-1 / 2}, \dot{\gamma}^{-1 / 2}$, of the neutralized poly(DEGSA) complex with $\frac{1}{4} \mathrm{Zn}^{2+}$ per chain at $70^{\circ} \mathrm{C}$. Open circles: unsheared sample; filled circles: sheared sample. The straight lines correspond to linear least squares fits. 


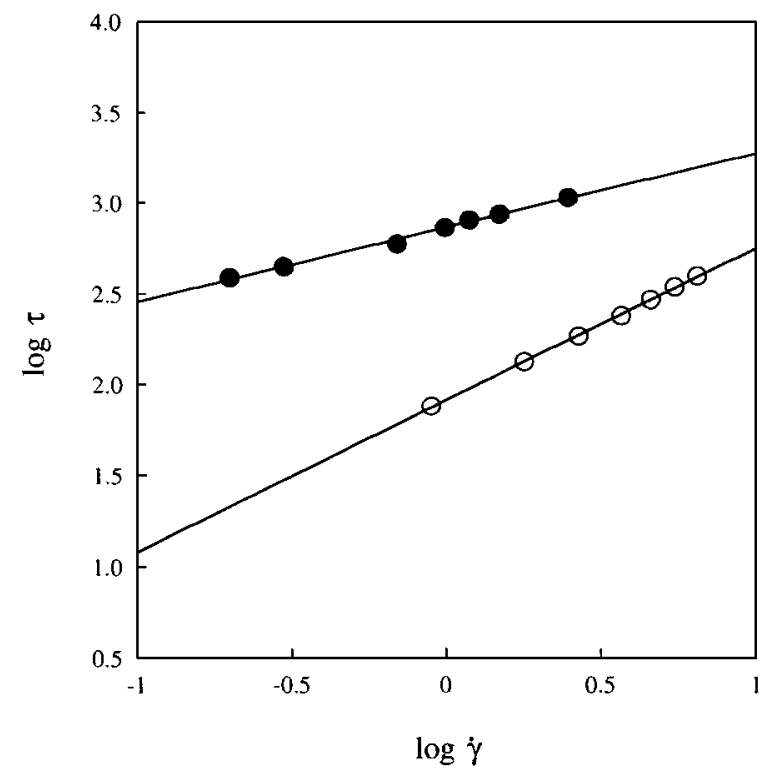

Figure 4. Log-log plot of the shear stress, $\tau$, vs. the shear rate, $\dot{\gamma}$, for the unsheared neutralized poly(DEG$\mathrm{SA}$ ) complex with $\frac{1}{4} \mathrm{Zn}^{2+}$ per chain at $30^{\circ} \mathrm{C}$ (open circles), and for the unsheared neutralized poly(DEG-SA) complex with $\frac{1}{2} \mathrm{Zn}^{2+}$ per chain at $70^{\circ} \mathrm{C}$ (filled circles). The lines were fit by assuming a power law relationship.

for the sample containing $\frac{1}{2} \mathrm{Zn}^{2+}$ per chain at $70^{\circ} \mathrm{C}$, respectively). Therefore, the neutralized poly (DEG$\mathrm{SA}$ ) complexes with $\mathrm{Zn}$ acetates show not only a thixotropic behavior, but also a shear thinning behavior.

\section{DISCUSSION}

In earlier work ${ }^{3}$ it was observed that the addition of small quantities (e.g., $3 \mathrm{w} / \mathrm{w} \%$ ) of various oxides of bivalent metals leads to drastic increases in the melt viscosity of various polyesters. These observations are similar to results obtained for ionomers, ${ }^{13,14}$ containing metal ions, in which the negatively charged monomer specii are copolymerized into the polymer chain. The increase of the viscosity of metal ion-containing coordination polyesters was attributed primarily to the presence of ionic links that are formed between the $\mathrm{Me}^{2+}$ and chain terminal $\sim \mathrm{COO}^{-}$groups. Coordination bonds between the empty coordination sites of the complexes ( see Schemes 1 and 2) and electron donor groups of the polymer chains resulted in the formation of a polarionic "telechelic" polymer network and in an increase in the effective molar mass of the polymer. ${ }^{15}$ To study the effect of metal carboxylate and metal carboxylato-hydroxo complexes on the viscous be- havior independently of the polymer chain ends, viscosity measurements of neutralized polyesters having alcoholic $\sim \mathrm{OH}$ groups at both chain ends with complexes of $\mathrm{Zn}^{2+}$ and $\mathrm{CH}_{3} \mathrm{COO}^{-}$were studied in the present work. The increase in the melt viscosity of the polyesters in the presence of the complexes is attributed to the formation of coordination bonds between electron donor groups within the polymer chain, and the empty coordination sites of the carboxylato-hydroxo complexes.

A strong thixotropic behavior and shear thinning was observed in viscosity measurements performed on the melts of neutralized polymers with different amounts of $\mathrm{Zn}^{2+}$ in the complex per one polymer chain. In other words, the melt viscosity of the neutralized, complexed polyesters studied was found to be dependent on both the rate and duration of shear. This observation is in agreement with results obtained in earlier work. ${ }^{4}$ When a constant shear is applied to these polymers, the network structure (which is a result of coordination bonds formed primarily between chain-internal electron donor groups and the $\mathrm{Zn}$-carboxylato-hydroxo complex) is progressively broken down. As a result, the melt viscosity decreases with time. This behavior is in contrast with results obtained on polyesters where the chain ends were not neutralized. In these polymers, the carboxylic chain ends of the macromolecules participate in forming the complexes if $\mathrm{MeO}$ is added to the polyester. These systems show very little shear thinning and virtually no thixotropy at comparable shear rates and at the time domain ranges used in this work. This contrast in the time- and shear ratedependent behavior is a result of the different complex structures in these two polymer systems.

It can be assumed that the carboxylato-hydroxo complex structure is formed primarily in the reaction between the equimolar amounts of acetic acid and $\mathrm{ZnO}$. Chain-internal electron donor groups of the neutralized poly (DEG-SA) such as ester carbonyl and chain-terminating $\sim \mathrm{OH}$ are primarily supposed to coordinate the unoccupied sites of the complex to form an ionic-polar network structure. The formation of the ionic coordination is due to iondipole interactions. This interaction force is expected to be weaker than the force between carboxylate groups and metal ions due to differences in the electron donor strengths. Thus, the external force (or shear stress) breaks down the complexed structure in the neutralized coordination polyesters at much lower shear stress values than for the metal ion-containing nonneutralized polyesters, which are coordinated to the central metal ion in the complex via carboxylate groups with stronger donacity. 
In the Casson-Asbeck plot, the angle formed by the two lines or the area of the triangle formed by the two lines and a vertical line through a given value for $\dot{\gamma}^{-1 / 2}$ is used as a criteria to estimate the degree of thixotropy of a fluid. ${ }^{9,10}$ A simple quantitative analysis showed that the angle or area of the sample with $\frac{1}{2} \mathrm{Zn}^{2+}$ per chain is higher than that of the sample with $\frac{1}{4} \mathrm{Zn}^{2+}$ per chain. This can be explained by the composition of the polymer complex system. It can be assumed that the number of structural linkages available for breaking during shearing in the sample with $\frac{1}{2} \mathrm{Zn}^{2+}$ per chain is higher than that in the sample with $\frac{1}{4} \mathrm{Zn}^{2+}$ per chain. The rate of breakdown of the structure during shearing at a given rate depends on the number of structural linkages available for breaking (see ref. 2). Thus, the expected degree of thixotropy of the melt with $\frac{1}{2} \mathrm{Zn}^{2+}$ per chain would be higher than that of the sample with $\frac{1}{4} \mathrm{Zn}^{2+}$ per chain. Our experiments showed exactly the same behavior (see the comparison of Figs. 2 and 3). It also can be concluded that the degree of thixotropy of the neutralized poly (DEG-SA) complexes with $\mathrm{Zn}$ acetates increases with the $\mathrm{Zn}^{2+}$ content.

Most thixotropic materials also show a shear thinning behavior. This can also be explained in terms of the molecular structure. In most random and highly entangled states, macromolecules have their greatest resistance to flow. As shear rates increase, polymer chains will begin to align in the shear field, reducing their resistance to flow past one another. However, in the case of thixotropy, as the fluid is subjected to a constant shear, the structures are progressively broken down and the melt viscosity decreases with time. Consequently, the shear stress decreases to maintain the constant shear rate.

Finally, it must be noted that, as mentioned in an earlier article, ${ }^{4}$ if the $\mathrm{Zn}$ acetate is not formed in situ in the polymer but is added after mixing an equivalent amount of $\mathrm{CH}_{3} \mathrm{COOH}$ and $\mathrm{ZnO}$, the original viscosity of the polymer remains unchanged, even after performing vigorous mixing and heat treatment over a long period. This can be interpreted as a result of high cohesive lattice energies for the acetate, which prevents the preformed salt from forming a dispersion in the polymer melt at the molecular level.

The authors are grateful to the Natural Sciences and Engineering Research Council (NSERC) of Canada for financial support, and are indebted to Ms. Anne Klemperer and Ms. Sandra de Jonge for their help with the preparation of the manuscript.

\section{REFERENCES AND NOTES}

1. J. D. Ferry, Viscoelastic Properties of Polymers, John Wiley, New York, 1980.

2. R. I. Tanner, Engineering Rheology, Clarendon, Oxford, 1988.

3. I. Vancsó-Szmercsányi and Z. Székely-Pécsi, J. Polym. Sci., Polym. Chem. Ed., 21, 1901 (1983).

4. I. Vancsó-Szmercsányi and P. Hischberg, Acta Chim. Acad. Sci. Hung., 83, 79 (1974).

5. A. Szilágyi, V. Izvekov, and I. Vancsó-Szmercsányi, J. Polym. Sci., Polym. Chem. Ed., 18, 2803 (1980).

6. I. Vancsó-Szmercsányi and A. Szilágyi, J. Thermal. Anal., 18, 235 (1980).

7. P. J. Flory, Principles of Polymer Chemistry, Cornell University Press, Ithaca, 1953, Chap. III.

8. I. Vancsó-Szmercsányi, B. White, and G. J. Vancso, J. Appl. Polym. Sci., Appl. Polym. Symp., 51, 241 (1992).

9. W. K. Asbeck, Off. Dig. Fed. Soc. Paint Technol, 33, 65 (1961).

10. P. E. Pierce, J. Paint Technol., 43, 35 (1971).

11. Brookfield guide to viscometer applications, AG7532, Brookfield Lab. Inc., Stoughton, MA, 1989.

12. N. Casson, Rheology of Disperse Systems, C. C. Mills (ed.), Pergamon, New York, 1959, p. 84.

13. L. Holliday, Ionic Polymers, Applied Science, London, 1975.

14. A. Eisenberg and M. King, Ion-Containing Polymers, Academic Press, New York, 1977.

15. B. White, G. Liu, I. Vancsó-Szmercsányi, and G. J. Vancso, Polym. Bull., 28, 95 (1992).

Received February 14, 1995

Revised July 28, 1995

Accepted August 3, 1995 